\title{
Cortical spreading depression occurs during elective neurosurgical procedures
}

\author{
Andrew P. Carlson, MD, MSCR, ${ }^{1}$ C. William Shuttleworth, PhD, ${ }^{2}$ Brittany Mead, BS, \\ Brittany Burlbaw, BS, ${ }^{1}$ Mark Krasberg, PhD, ${ }^{1}$ and Howard Yonas, MD ${ }^{1}$ \\ Departments of ${ }^{1}$ Neurological Surgery and ${ }^{2}$ Neurosciences, University of New Mexico School of Medicine, Albuquerque, \\ New Mexico
}

\begin{abstract}
OBJECTIVE Cortical spreading depression (CSD) has been observed with relatively high frequency in the period following human brain injury, including traumatic brain injury and ischemic/hemorrhagic stroke. These events are characterized by loss of ionic gradients through massive cellular depolarization, neuronal dysfunction (depression of electrocorticographic [ECoG] activity) and slow spread (2-5 mm/min) across the cortical surface. Previous data obtained in animals have suggested that even in the absence of underlying injury, neurosurgical manipulation can induce CSD and could potentially be a modifiable factor in neurosurgical injury. The authors report their initial experience with direct intraoperative ECOG monitoring for CSD.
\end{abstract}

METHODS The authors prospectively enrolled patients undergoing elective craniotomy for supratentorial lesions in cases in which the surgical procedure was expected to last $>2$ hours. These patients were monitored for CSD from the time of dural opening through the time of dural closure, using a standard $1 \times 6$ platinum electrode coupled with an $A C$ or full-spectrum DC amplifier. The data were processed using standard techniques to evaluate for slow potential changes coupled with suppression of high-frequency ECoG propagating across the electrodes. Data were compared with CSD validated in previous intensive care unit (ICU) studies, to evaluate recording conditions most likely to permit CSD detection, and identify likely events during the course of neurosurgical procedures using standard criteria.

RESULTS Eleven patients underwent ECoG monitoring during elective neurosurgical procedures. During the periods of monitoring, 2 definite CSDs were observed to occur in 1 patient and 8 suspicious events were detected in 4 patients. In other patients, either no events were observed or artifact limited interpretation of the data. The DC-coupled amplifier system represented an improvement in stability of data compared with AC-coupled systems. Compared with more widely used postoperative ICU monitoring, there were additional challenges with artifact from saturation during bipolar cautery as well as additional noise peaks detected.

CONCLUSIONS CSD can occur during elective neurosurgical procedures even in brain regions distant from the immediate operative site. ECoG monitoring with a DC-coupled full-spectrum amplifier seemed to provide the most stable signal despite significant challenges to the operating room environment. CSD may be responsible for some cases of secondary surgical injury. Though further studies on outcome related to the occurrence of these events is needed, efforts to decrease the occurrence of CSD by modification of anesthetic regimen may represent a novel target for study to increase the safety of neurosurgical procedures.

http://thejns.org/doi/abs/10.3171/2015.11.JNS151871

KEY WORDS neurosurgical procedures; intraoperative monitoring; cortical spreading depolarization; cortical spreading depression; diagnostic and operative techniques

ORTICAL spreading depressions (CSDs) are waves of near-complete cellular depolarization that spread slowly across the cortical surface at a rate of $2-5$ $\mathrm{mm} / \mathrm{min}$ and result in transient loss of cell signaling capabilities. This results in the observed "depression" of elec- trocorticographic (ECoG) activity. ${ }^{13}$ While CSD has been thought to represent the physiological underpinnings of brain dysfunction during migraine aura for many years, ${ }^{15}$ the significance of these events for brain injury and stroke has become apparent only relatively recently. Cooperative

ABBREVIATIONS AVM = arteriovenous malformation; $C S D=$ cortical spreading depression; $E C O G=$ electrocorticographic; $I C U=$ intensive care unit; $O R=$ operating room . SUBMITTED August 14, 2015. ACCEPTED November 25, 2015.

INCLUDE WHEN CITING Published online March 11, 2016; DOI: 10.3171/2015.11.JNS151871. 
studies of intensive care unit (ICU) patients have definitively documented the presence of CSD after a variety of types of acute brain injury including traumatic brain injury, subarachnoid hemorrhage, intracerebral hemorrhage, and ischemic stroke. ${ }^{12,16}$ These events likely have a cyclical role in the progression of brain injury. The occurrence of brain injury induces CSD, ${ }^{9}$ and the occurrence of CSD, particularly in at-risk tissue (such as ischemic penumbra) results in further expansion of injury due to the massive metabolic requirements placed on the brain to restore ionic gradients. Several lines of evidence support this assertion, including observed expansion of malignant stroke with repeated CSD,${ }^{14}$ association with delayed cerebral ischemia (DCI) after subarachnoid hemorrhage, ${ }^{7}$ progressive metabolic dysfunction with repeated events, ${ }^{3}$ and association with worse clinical outcomes. ${ }^{8}$

We recently hypothesized that CSD may occur during elective neurosurgical procedures based on physiological plausibility and an animal model of neurosurgical procedures. ${ }^{4}$ Based on the observed deleterious effect of CSD after brain injury, we hypothesize that this may be a factor contributing to otherwise poorly explained surgery-related brain injury. In this study, we report our initial prospective observations with monitoring of ECoG activity during elective neurosurgical procedures.

\section{Methods}

Patients requiring elective neurosurgical resection of intracranial tumors were prospectively recruited for this observational study. Local institutional review board approval was obtained. Entry criteria included the following: 1) patient had no previous acute hemorrhage, infarct, or other event that could be considered acute brain injury prior to the procedure, 2) patient required an extensive enough procedure that at least 2 hours of monitoring time were expected to obtain adequate data, and 3) placement of an electrode strip was not thought to interfere with the neurosurgical procedure. Informed consent for monitoring was obtained from all individuals.

Once the craniotomy was performed, a small durotomy was created and a $1 \times 6$ platinum electrode strip (Auragen, Integra Epilepsy) was placed in a position in the subdural space radiating away from the opening so as not to interfere with the surgical procedure. This positioning placed the strip over an area of healthy brain away from the pathological process. The distance from the site of direct surgical manipulation could vary significantly, since in convexity lesions the strip was within $2-3 \mathrm{~cm}$ of the surgical site whereas in skull base lesions, the strip was on the convexity surface and could be $6 \mathrm{~cm}$ or more from the lesion. In initial AC recordings, a differential montage was used for acquisition, whereas in the DC recording group, a reference electrode was placed on a stable part of the scalp away from the incision prior to the surgical procedure and data were acquired based on this reference. A standard EEG cup electrode was glued to the scalp using collodion and filled with conductive jelly for the reference. $\mathrm{An} \mathrm{Ag}$ / $\mathrm{AgCl}$ grounding electrode was placed on the patient's shoulder. The electrodes were attached to one of two systems. In the initial data using the AC-coupled system, a full-spectrum AC-coupled bioamplifier was attached to a PowerLab system (ADInstruments) as is standard for postoperative monitoring of CSD. ${ }^{12,16}$ As technology improved for ICU monitoring, we began using a modified DCcoupled, full-spectrum research amplifier connected to a Component Neuromonitoring System (Moberg Research Inc.). This system allows for better detection of the slow potential changes in the DC signal characteristic of CSD.

Data were then uploaded to a central server and analyzed using LabChart software (ADInstruments). Both the raw referential data, as well as postprocessed differential recordings, were analyzed; however, due to significant reduction in artifact, the differential recordings were preferred for data analysis. These differential recordings were constructed by subtracting the signal from the adjacent channel (i.e., 1-2, 2-3, etc...). These signals were then filtered at $0.5-50 \mathrm{~Hz}$ to remove low-frequency fluctuations as well as noise of $60 \mathrm{~Hz}$ or higher that was noted to be prominent in the operating room (OR). To quantify these changes, fast Fourier transformations were performed to identify noise peaks for filtering thresholds. In addition, the periods of amplifier saturation after bipolar cautery were compared. This was done by identifying times of artifact consistent with bipolar cautery. The length of time that the high-frequency signal was lost was then measured. Only leads and events where there was recovery of the baseline high-frequency signal were used.

Data were reviewed for CSD as previously defined from ICU studies. ${ }^{10,16}$ Because of the higher than usual degree of DC signal fluctuation due to surgical movement, particularly close attention was paid to the more stable filtered signal to evaluate for propagating suppression. The 60 -second integral of the filtered signal was also used, as has been previously suggested, ${ }^{14}$ to aid in detection of events. Events were scored by 2 reviewers experienced in evaluation of CSD (A.C. and C.W.S.). CSD was defined as a slow shift in the DC signal on 2 or more adjacent electrodes accompanied by suppression and subsequent recovery of the high-frequency signal. In addition to definite events, suspicious events that fulfilled those criteria but had more abnormal DC shifts or some degree of artifact were also recorded. Definite and suspicious events were recorded and only agreed upon by consensus of the scorers.

Basic demographic data, including type of surgical procedure, diagnosis, and postoperative clinical course as well as new radiographic findings, were obtained for each patient in the group of patients who were monitored with the DC system. In the initial exploratory phase, only monitoring was performed anonymously as part of the institutional review board request.

\section{Results}

\section{Summary of CSD Detection}

Basic data regarding the subjects are listed in Table 1. Overall, 11 patients underwent electrophysiological monitoring during elective neurosurgical procedures. Three of these were monitored using an AC-coupled system, whereas the remaining 8 patients were monitored using a DC system. One patient undergoing transcranial resection 


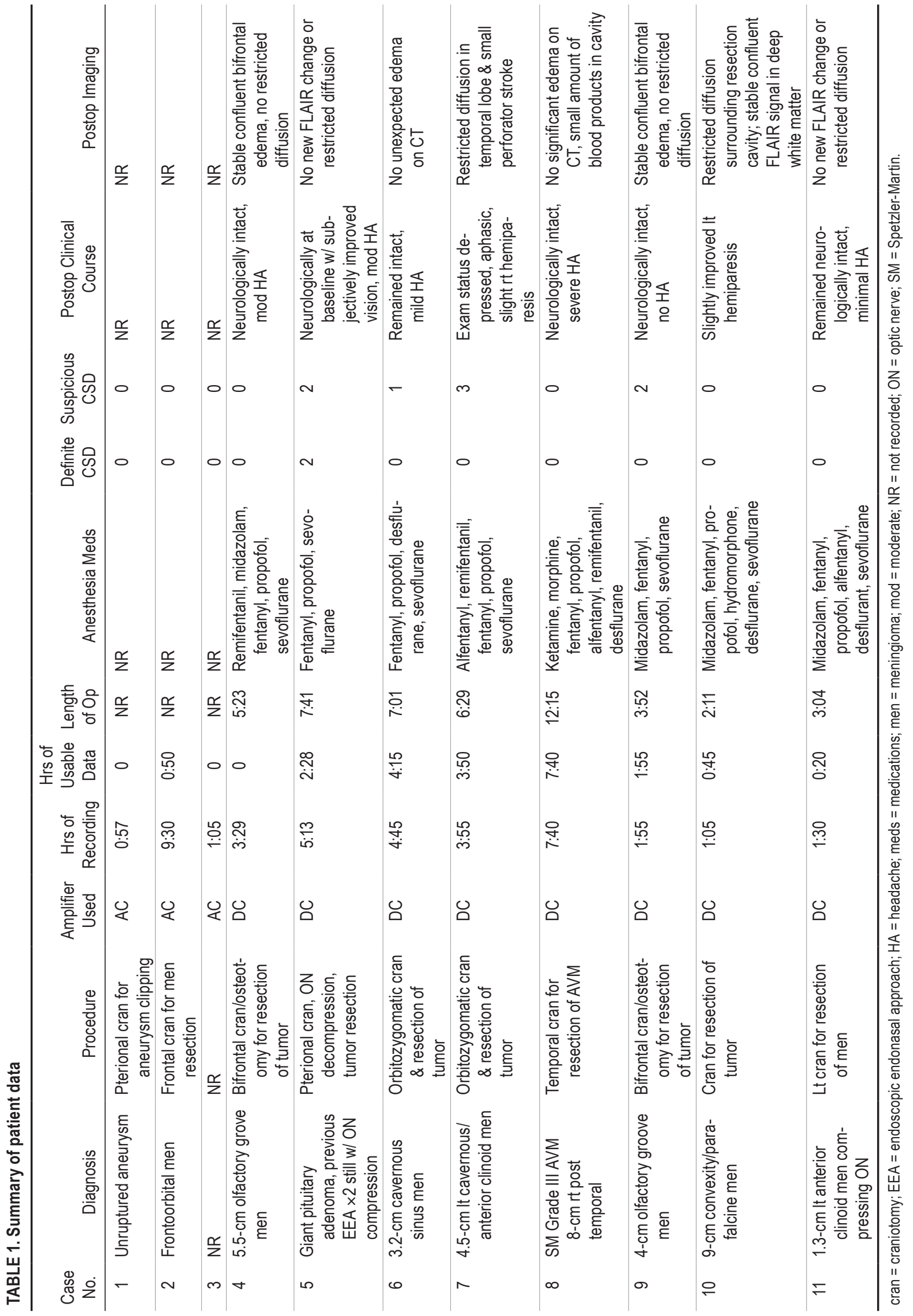


of residual giant pituitary adenoma was found to have 2 definite CSDs (Fig. 1). Eight suspicious events occurred in 4 patients (Table 1). All patients in whom events were noted were monitored with the DC amplifier. In 1 patient postoperative deterioration was noted on examination after she underwent an orbitozygomatic craniotomy for a $4.5-\mathrm{cm}$ medial sphenoid wing/cavernous meningioma, which was attached to the middle cerebral artery vasculature. Postoperatively, she was found to have significant edema in the temporal lobe, as well as a perforator infarct coupled with mild hemiparesis and aphasia. This patient had 3 suspicious events, though significant artifact limited establishing these events as definite. Due to the small numbers, no attempt to correlate the occurrence of events with radiographic or clinical outcome in this initial study was made.

\section{Characteristics of Patient With Clearly Identified CSD}

Specific intraoperative data for the single patient with clearly identified CSD are summarized in Fig. 2. This includes the temporal profile during surgery with 1 event (definite) occurring near the beginning, and 3 additional events occurring later in the monitoring period. This patient had previously presented with vision loss related to a giant pituitary adenoma. The patient had previously undergone 2 endoscopic transsphenoidal debulking procedures and was taken in for a craniotomy for optic nerve decompression and resection. The electrode strip was placed on the superficial frontal lobe and a standard subfrontal/transsylvian approach was used for optic nerve decompression and tumor resection. In Fig. 2 intraoperative video screenshots show the strip being placed posteriorly under the bone edge on the frontal lobe. Due to extensive

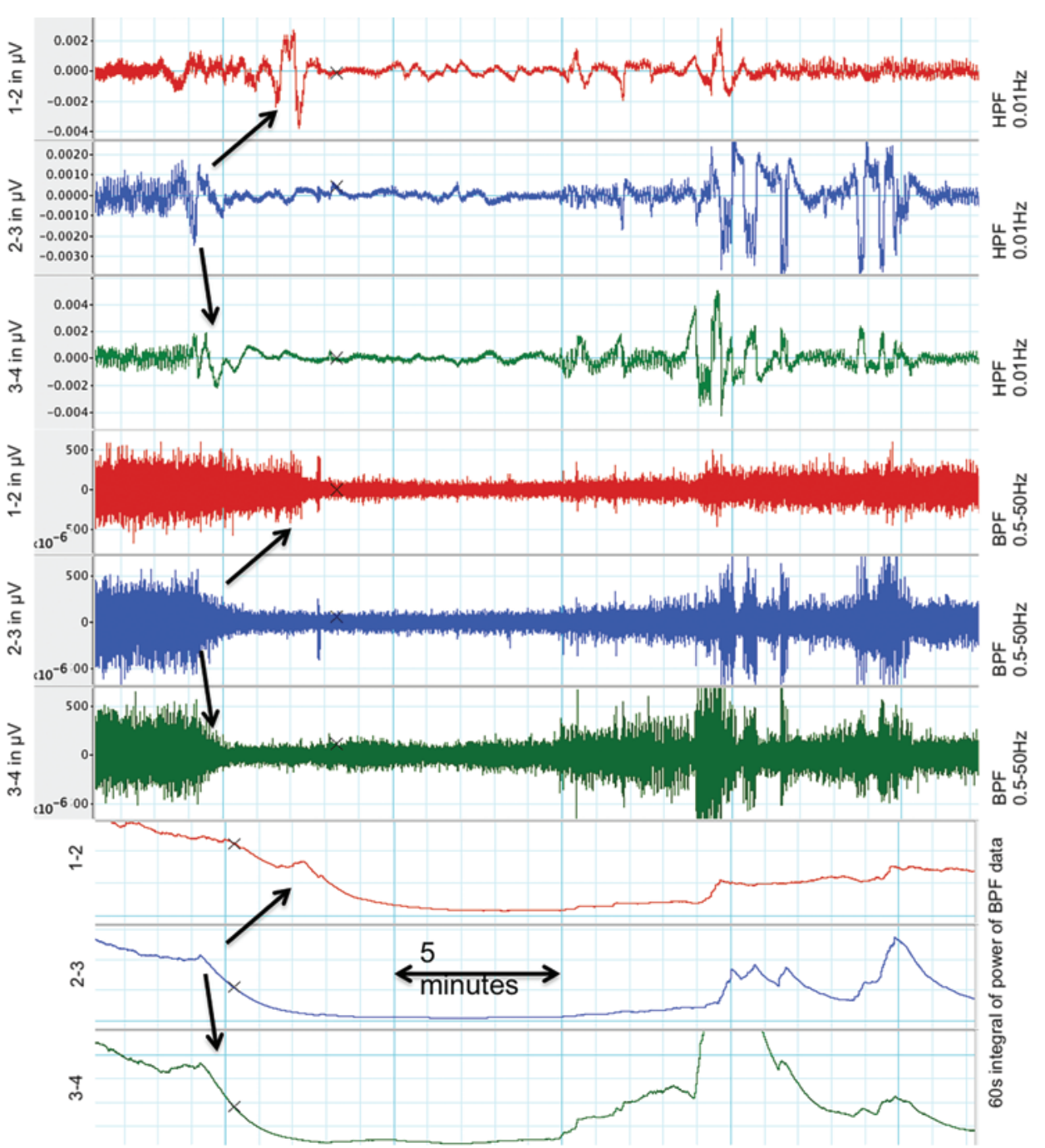

FIG. 1. Electrocorticography tracings of three differential lead (1-2, 2-3, and 3-4) signals involved in CSD in a patient undergoing resection of a giant pituitary adenoma. The top three traces represent the raw DC signal filtered at $0.01 \mathrm{~Hz}$ to remove drift. A shift in the DC potential is noted, spreading from Lead 2 to Leads 3 and 1 . The middle three traces show the data at $0.5-50 \mathrm{~Hz}$. Clear suppression of the ECoG activity is noted with slow recovery over the subsequent 10-15 minutes. The bottom three traces show the 60 -second integral of the power of the ECoG signal. A characteristic falloff is noted coinciding with the ECoG suppression (arrows). $\mathrm{BPF}=$ band pass filtered; HPF = high pass filter. 
adhesion to the optic apparatus and surrounding vasculature, the operation required more time than expected, but there were no concerning intraoperative findings such as vascular injury or bleeding. No significant fluctuation was observed in systemic variables or maintenance anesthetic (Fig. 2). The patient recovered from surgery uneventfully and had improvement in visual fields and no new deficit.

\section{Technical Approaches to CSD Detection in the OR Environment}

Data characteristics were evaluated to address the specific challenges of the OR environment. Fast Fourier transformation was performed for the raw data sets and results were compared with representative data sets obtained from standard postoperative monitoring in patients with acute brain injury. Representative data are displayed in Fig. 3. In addition to standard electrical $60-\mathrm{Hz}$ noise, multiple additional noise peaks were noted, particularly at 92 and $96 \mathrm{~Hz}$. Eliminating these peaks with band pass filtering improved the signal-to-noise ratio.

In addition, significant artifact was noted with amplifier saturation during bipolar cautery when using the $\mathrm{AC}$ system. This saturation led to a consistent refractory period in which no ECoG could be obtained. Exploratory bench testing revealed that the newly acquired DC system used for ICU monitoring markedly decreased the refractory period from cautery. Using the new recording system, we compared the data quality near bipolar cautery times. In the AC data, we identified 62 single-lead events with loss of signal during bipolar cautery and subsequent restoration of high-frequency signal (mean length of loss of signal $42.4 \pm 23.8$ seconds $( \pm$ SD). No refractory period could be identified in the DC data after cauterization (see Fig. 4).

\section{Discussion}

These data represent the first observations of CSD oc-

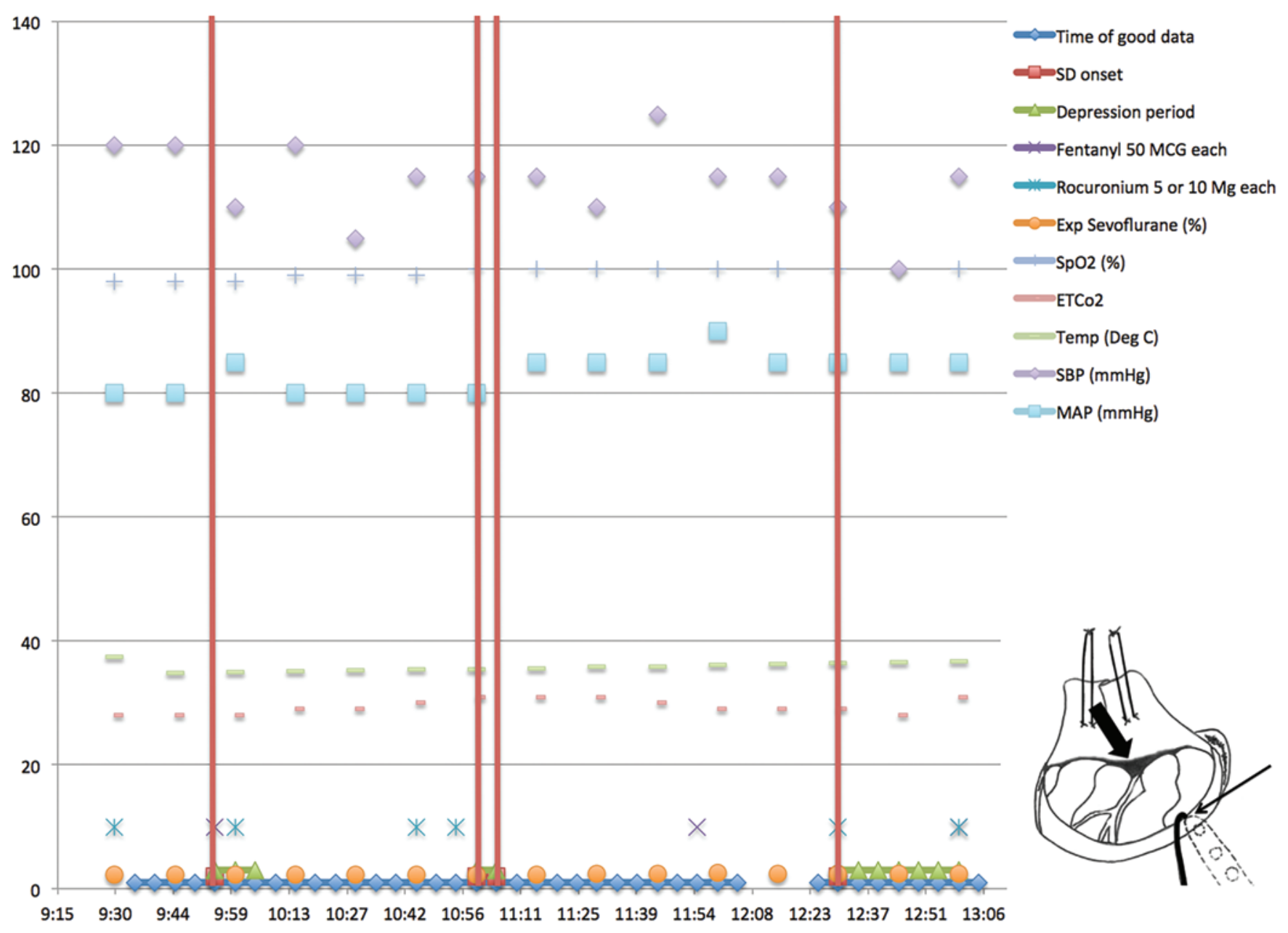

FIG. 2. Intraoperative physiological variables show stability of standard variables during the monitored portion of the surgical procedure in the patient described in Fig. 1. The y axis is a common scale for all numeric variables with units shown in the key for each variable. Timing of drug administration and timing of monitoring are shown in the region $<20$. The vertical red lines show the times of definite and suspicious $\mathrm{CSD}$. ETCO2 = end tidal $\mathrm{CO}_{2} ; \mathrm{Exp}=$ expiratory; $\mathrm{MAP}=$ mean arterial pressure; $\mathrm{SBP}=$ systolic blood pressure; $\mathrm{SD}=$ spreading depression; $\mathrm{SpO} 2$ = capillary oxygen saturation. The lower right image is a sketch of the surgical procedure. A left pterional craniotomy is shown with the electrode strip placed under the dural edge (narrow arrow). The surgical procedure was centered down the pterional corridor in a subfrontal direction (wide arrow). 


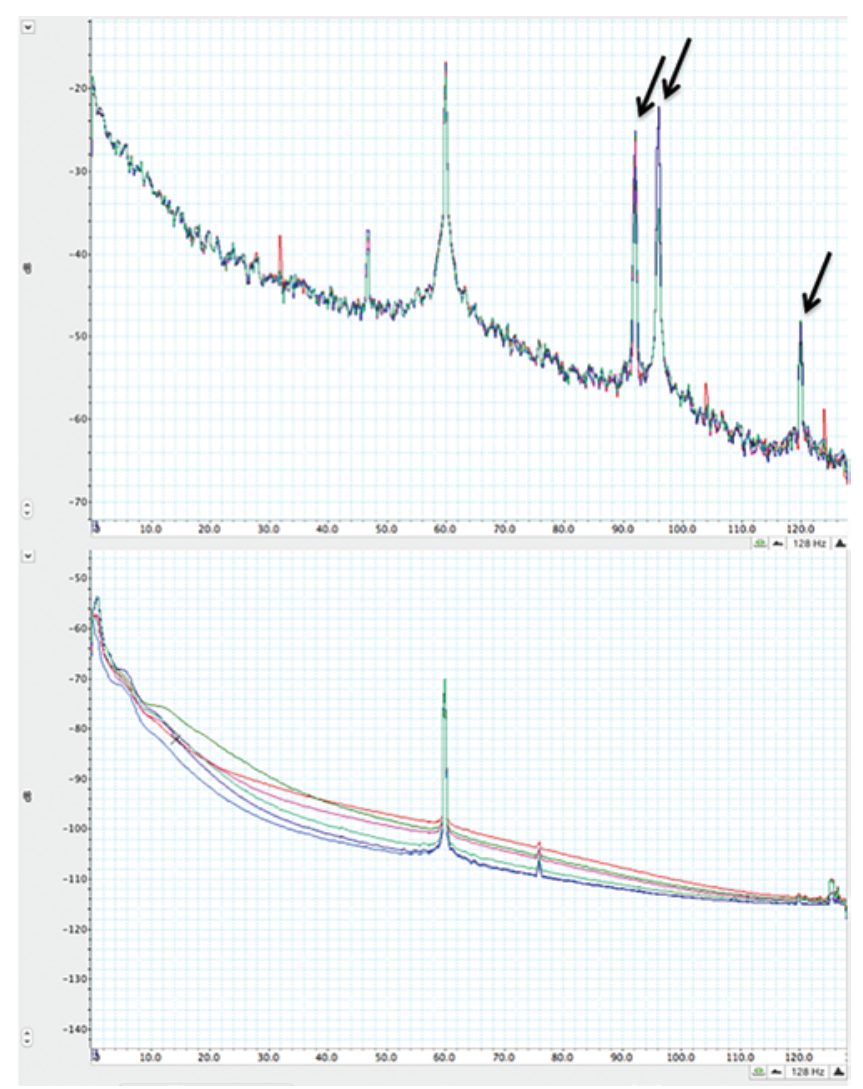

FIG. 3. Fast Fourier transformation (FFT) of intraoperative data (upper) compared with ICU-acquired data (lower). The upper graph shows the averaged signal of 9442 FFTs for each of the 6 electrodes (overlapping colors). The arrows point out the large peaks of noise not noted in the recordings from the ICU (lower). The lower graph shows the averaged data of 27,720 FFTs, again for each of the six electrodes shown as separate colors. Figure is available in color online only.

curring during elective neurosurgical intervention. Based on our previous observations in animal studies, ${ }^{4}$ we hypothesized that even standard neurosurgical manipulation such as bipolar cauterization and brain retraction might result in CSD even when patients are under general anesthesia. The present preliminary observations support this hypothesis with definite CSD occurring in 1 patient and suspicious CSD in 4 others. This observation will require further confirmatory data and more widespread monitoring to better evaluate whether the occurrence, frequency, or subtype of CSD (such as clusters or isoelectric depolarizations) are associated with worse outcomes. In the case of acute brain injury, it is clear that frequency and severity of CSD are correlated with worse clinical and radiographic outcome in traumatic brain injury, stroke, and subarachnoid hemorrhage, ${ }^{7,8,14}$ and thus it is possible that a parallel association in the case of surgical brain injury exists.

Surgery-related brain injury is a concept that has been discussed previously, but because of the very heterogeneous nature of injury types, it has not been well studied as a specific entity. Many have suggested that overaggressive retraction of the brain may contribute to these injuries. ${ }^{1}$ Other factors such as venous obstruction, direct injury, or arterial stroke may also result in postoperative clinical and/or radiographic deterioration. CSD may represent a new pathophysiological mechanism for poorly explained surgery-induced brain injury that does not have a clearly identifiable cause (such as mechanical contusion).

These mechanisms are critical to explore because, despite dramatic advances in microsurgical technique that have improved the safety of neurosurgical procedures, there are still patients with complex cranial pathology who have an unexpectedly poor postoperative course and who may benefit from targeted therapy. Mounting data suggest that CSD may be a modifiable risk factor in cases of acute brain injury ${ }^{11,17}$ and may be a target to improve the safety of neurosurgical procedures in general. A critical difference in cases of elective neurosurgery is that there is a potential opportunity for prophylactic intervention. There are only a few drugs currently suggested to suppress CSD in the case of acute brain injury including ketamine ${ }^{11}$ and nimodipine; 5,6 however, there are multiple medications thought to prevent CSD in the case of migraine aura. ${ }^{2} \mathrm{An}$ approach involving premedication in surgical procedures might therefore be a rational hypothesis to test.

Our study design sought to carefully eliminate patients with acute brain injury such as stroke or hemorrhage, and the monitoring strips were placed remotely from the site of direct surgery for practical reasons. Because CSD events were observed at all, we think that this observation likely represents only the "tip of the iceberg." It is probable that there is significant sampling bias in our data and that CSD events occur more frequently than we observed in closer proximity to the site of surgical manipulation. In addition, because only a relatively short period of observation could be attained, we may have missed additional events occurring near the beginning or the end of the procedure. In some cases, extensive artifact from cautery, movement, or other sources interfered with adequate data collection. The lesions were nearly all extraaxial as well, which did not require direct corticectomy, which would be expected to be more likely to induce CSD. The one exception to this was the patient with a large arteriovenous malformation (AVM). In this case, no CSD was observed but this may have been related to two factors: first, there was extensive bipolar artifact; second, our anesthesiology colleagues, who had become interested in the developing data regarding CSD and based on the preliminary observations of Hertle et al.," ${ }^{11}$ administered anesthetic doses of ketamine (which has been strongly implicated in the suppression of CSD) to the patient as part of their anesthetic plan.

We have tried to document the blood flow response evident with CSD by using laser speckle imaging, as was used in cases of malignant hemispheric stroke by Woitzik et al., ${ }^{18}$ but found this to be impractical due to the need for direct imaging of the cortex without manipulation. In our relatively short periods of observation (5-15 minutes), we did not detect any CBF changes consistent with CSD (unpublished personal observations).

Based on our observations, further study of CSD in the OR environment must account for the inherent additional monitoring challenges in the OR including additional electrical noise and electrocautery artifact. We have found that the most stable signal is achieved using the aforementioned techniques that involve a full-spectrum DC ampli- 


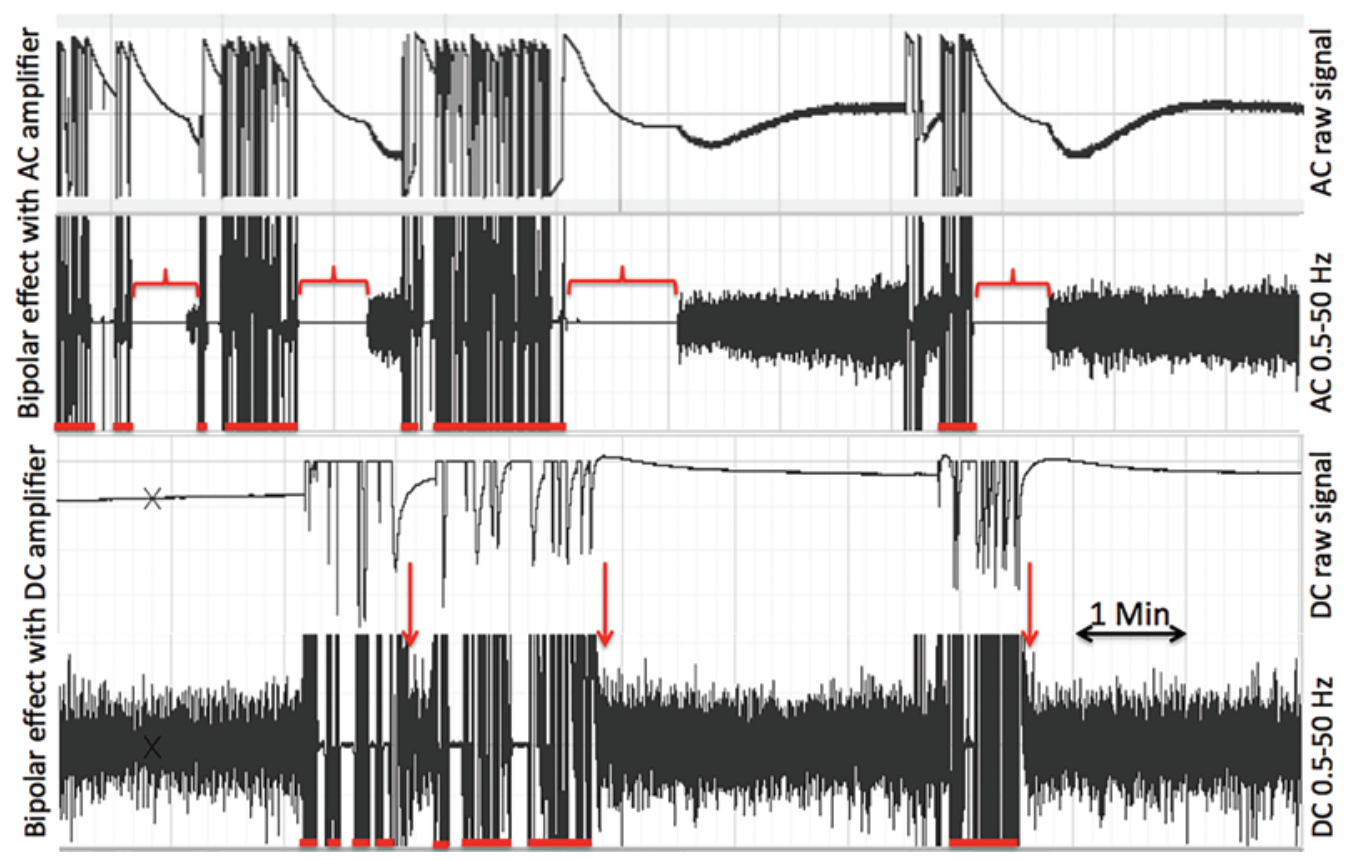

FIG. 4. Comparison of intraoperative signals obtained using an AC-coupled system (upper) and using a DC-coupled system (lower) in response to bipolar cautery during tumor resection. The horizontal red lines below each data set show the times of cauterization. The raw signal is shown above the filtered data for each example. Note the refractory period after cautery seen in the top traces (red brackets) due to amplifier saturation compared with the lack of refractory period in the bottom traces (red arrows). Figure is available in color online only.

fier with monopolar data acquisition and bipolar processing. The standard AC-coupled amplifiers in clinical use for ICU monitoring are likely too sensitive to bipolar artifact to be reliable in the OR environment.

We think that these acquisition techniques allowed for the detection of CSD when previous studies of cortical monitoring for epilepsy have not. This conclusion has previously been made to explain the nondetection of CSD in conventional long-term epilepsy monitoring. ${ }^{12}$ First, the range of the amplifier has to be broad enough to allow for detection of the slow potential changes, and most clinical amplifiers are limited to the high-frequency range of ECoG activity. The DC amplifier we used offers a true full-spectrum acquisition. Second, these events move so slowly $(2-5 \mathrm{~mm} / \mathrm{min})$ that they can only be observed with a very compressed time scale, which is not typical during epilepsy monitoring and cannot be assessed with most commercial programs.

There are clearly limitations to our study. The study represents a very preliminary observation, and definite CSD was only demonstrated in 1 patient, possibly related to the reasons listed above. Because of the distance from the site of direct surgical manipulation in most cases, it is impossible to link the observed events to a specific manipulation. While we suspect that surgical manipulation was causative in the observed CSD, we can only definitely conclude that these events occur. Because we do not have reliable methods for prolonged noninvasive monitoring for CSD, we cannot definitely say that the events would not have occurred in the absence of surgical manipulation, although this seems unlikely. In addition, our clinical hypothesis of CSD's deleterious effect was not supported by our current small data set in that the patient with CSD had a very benign clinical and radiographic outcome, whereas 1 patient who experienced a postoperative stroke did not exhibit any definite events. This patient did, however, have 3 suspicious CSD events during the observation period, and monitoring during the immediate postoperative period may have been informative in both of these patients. Larger data sets with postoperative monitoring in highrisk cases are clearly needed to better answer this question.

\section{Conclusions}

Cortical spreading depression occurs during elective neurosurgical procedures. The use of continuous ECoG monitoring using a DC-coupled system during the period of dural opening seems to offer a reasonable platform for monitoring in the OR. The true incidence of and risk factors for CSD and its effect on clinical outcome remain unknown. Based on animal studies and clinical data from patients with brain injury, it is possible that recurrent CSD is associated with worse clinical outcomes and may be a novel explanation for otherwise poorly understood surgery-induced brain injury. Further studies are required to determine the relationship between intraoperative CSD and clinical outcome to allow the design of trials to mitigate the deleterious effects of these events.

\section{Acknowledgments}

We thank Michael Carey, MD, for assistance with initial intraoperative data collection attempts, and we would like to dedicate this article to his memory. 
This study was supported through internal funding from the Department of Neurosurgery at the University of New Mexico.

\section{References}

1. Andrews RJ, Bringas JR: A review of brain retraction and recommendations for minimizing intraoperative brain injury. Neurosurgery 33:1052-1064, 1993

2. Ayata C, Jin H, Kudo C, Dalkara T, Moskowitz MA: Suppression of cortical spreading depression in migraine prophylaxis. Ann Neurol 59:652-661, 2006

3. Bosche B, Graf R, Ernestus RI, Dohmen C, Reithmeier T, Brinker G, et al: Recurrent spreading depolarizations after subarachnoid hemorrhage decreases oxygen availability in human cerebral cortex. Ann Neurol 67:607-617, 2010

4. Carlson AP, Carter RE, Shuttleworth CW: Vascular, electrophysiological, and metabolic consequences of cortical spreading depression in a mouse model of simulated neurosurgical conditions. Neurol Res 34:223-231, 2012

5. Dietz RM, Weiss JH, Shuttleworth CW: $\mathrm{Zn}^{2+}$ influx is critical for some forms of spreading depression in brain slices. $\mathbf{J}$ Neurosci 28:8014-8024, 2008

6. Dreier JP, Windmüller O, Petzold G, Lindauer U, Einhäupl KM, Dirnagl U: Ischemia triggered by red blood cell products in the subarachnoid space is inhibited by nimodipine administration or moderate volume expansion/hemodilution in rats. Neurosurgery 51:1457-1467, 2002

7. Dreier JP, Woitzik J, Fabricius M, Bhatia R, Major S, Drenckhahn C, et al: Delayed ischaemic neurological deficits after subarachnoid haemorrhage are associated with clusters of spreading depolarizations. Brain 129:3224-3237, 2006

8. Hartings JA, Bullock MR, Okonkwo DO, Murray LS, Murray GD, Fabricius $M$, et al: Spreading depolarisations and outcome after traumatic brain injury: a prospective observational study. Lancet Neurol 10:1058-1064, 2011

9. Hartings JA, Rolli ML, Lu XC, Tortella FC: Delayed secondary phase of peri-infarct depolarizations after focal cerebral ischemia: relation to infarct growth and neuroprotection. $\mathbf{J}$ Neurosci 23:11602-11610, 2003

10. Hartings JA, Watanabe T, Dreier JP, Major S, Vendelbo L, Fabricius M: Recovery of slow potentials in AC-coupled electrocorticography: application to spreading depolarizations in rat and human cerebral cortex. J Neurophysiol 102:2563-2575, 2009

11. Hertle DN, Dreier JP, Woitzik J, Hartings JA, Bullock R, Okonkwo DO, et al: Effect of analgesics and sedatives on the occurrence of spreading depolarizations accompanying acute brain injury. Brain 135:2390-2398, 2012
12. Lauritzen M, Dreier JP, Fabricius M, Hartings JA, Graf R, Strong AJ: Clinical relevance of cortical spreading depression in neurological disorders: migraine, malignant stroke, subarachnoid and intracranial hemorrhage, and traumatic brain injury. J Cereb Blood Flow Metab 31:17-35, 2011

13. Leao AA: Further observations on the spreading depression of activity in the cerebral cortex. J Neurophysiol 10:409414,1947

14. Nakamura H, Strong AJ, Dohmen C, Sakowitz OW, Vollmar S, Sué M, et al: Spreading depolarizations cycle around and enlarge focal ischaemic brain lesions. Brain 133:1994-2006, 2010

15. Olesen J, Larsen B, Lauritzen M: Focal hyperemia followed by spreading oligemia and impaired activation of $\mathrm{rCBF}$ in classic migraine. Ann Neurol 9:344-352, 1981

16. Strong AJ, Fabricius M, Boutelle MG, Hibbins SJ, Hopwood SE, Jones R, et al: Spreading and synchronous depressions of cortical activity in acutely injured human brain. Stroke 33:2738-2743, 2002

17. Strong AJ, Hartings JA, Dreier JP: Cortical spreading depression: an adverse but treatable factor in intensive care? Curr Opin Crit Care 13:126-133, 2007

18. Woitzik J, Hecht N, Pinczolits A, Sandow N, Major S, Winkler MK, et al: Propagation of cortical spreading depolarization in the human cortex after malignant stroke. Neurology 80:1095-1102, 2013

\section{Disclosures}

The authors report no conflict of interest concerning the materials or methods used in this study or the findings specified in this paper.

\section{Author Contributions}

Conception and design: Carlson, Shuttleworth, Yonas. Acquisition of data: Carlson, Mead, Burlbaw, Krasberg. Analysis and interpretation of data: Carlson. Drafting the article: Carlson. Critically revising the article: Shuttleworth, Yonas. Reviewed submitted version of manuscript: all authors. Approved the final version of the manuscript on behalf of all authors: Carlson. Administrative/technical/material support: Mead, Burlbaw.

\section{Correspondence}

Andrew P. Carlson, Department of Neurological Surgery, University of New Mexico School of Medicine, MSC10 5615, Albuquerque, NM 87131. email: andrewcarlson@salud.unm.edu. 